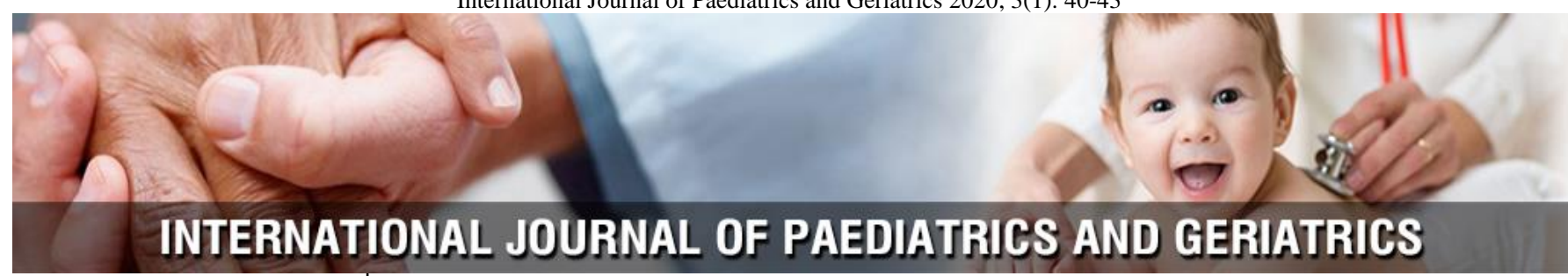

P-ISSN: 2664-3685

E-ISSN: 2664-3693

www.paediatricjournal.com

IJPG 2020; 3(1): 40-43

Received: 22-11-2019

Accepted: 26-12-2019

Abdul Sattar Ibrahim

Mohammed

Pediatric Consultant, Central

Child Teaching Hospital,

Baghdad, Iraq

Bashar Ibrahim Mohammed

Alamria Hospital Department

of emergency Medicine,

Alanbar, Iraq

Mohammad Ali Mohammad

Central Child Teaching

Hospital, Baghdad, Iraq
Corresponding Author:

Abdul Sattar Ibrahim

Mohammed

Pediatric Consultant, Central

Child Teaching Hospital,

Baghdad, Iraq

\section{Febrile neutropenia in children with acute leukemia receiving chemotherapy; Clinical, laboratory characteristics and outcome}

\author{
Abdul Sattar Ibrahim Mohammed, Bashar Ibrahim Mohammed and \\ Mohammad Ali Mohammad
}

DOI: https://doi.org/10.33545/26643685.2020.v3.i1a.59

\begin{abstract}
Background: Fever and neutropenia among the most common and serious complications of cancer chemotherapy. The diagnosis of sepsis in children with febrile neutropenia remain difficult due to nonspecific clinical and laboratory signs of infect-ion, so hospitalization and empirical intravenous broad spectrum antibiotic should be initiated as early as possible. The aim of this prospective study is to describe clinical, labora-tory characteristic and outcome of febrile neutropenic episodes.

Methods: One hindered seventy febrile neutropenic episodes observed in 100 children with acute leukemia receiving chemotherapy in the oncology department of child's central teaching hospital /Baghdad over a period of 10 months (from march through December 2007).

Results: febrile neutropenia was frequent complication, and severe neutropenia (ANC $<200 \mathrm{cell} / \mathrm{mm}$ ) was encountered in 47 episodes (27\%) of total episodes. Clinical signs and symptoms suggestive of infection were evident in $38 \%$ of neutropenic episodes, the respiratory system was the most frequently affected site encountered in 17\%. Microbiological documented infection found in $16 \%(\mathrm{n}=27)$ of episodes all from local sites other than blood, bacteremia couldn't be detected in any samples. G-ve bacilli were the most frequently isolated $(63 \%)$ followed by G+ve microorganisms in $(37 \%)$. Fatality rate was $24 \%$ of total patients.

Conclusion: This study confirmed that our laboratory is behind the global standard for isolation of causative organisms.
\end{abstract}

Keywords: Fever, fatality, episodes

\section{Introduction}

Patients with hematologic disorder are complications as a consequence of perturbation of one or more components of their host defense system ${ }^{[1]}$. Predisposed to variety of infectious Neutropenia remain the major defect for many patients and therefore continues to serve as a model system for dealing with infections in patients who are immuno compromised [2]. About $1 / 2$ of neutropenic patients who become febrile have an established or occult infection and at least $1 / 5$ of patients with neutrophil count $<100$ cells $/ \mathrm{mm}^{[3]}$ have bacteremia ${ }^{3}$.In mid1980 the spectrum of bacteria causing infection began to change with steady increase in G+ve infection occurred until presently $60-70 \%$ of bacreremias ${ }^{[19]}$ with Coag.-ve staph \&staph. Aureus are the predominant. Although the goal of initial evaluation is to identify potential sources of infection, this will not be successful in the majority of patients ${ }^{[4]}$.

\section{Aims of study}

To study febrile episodes in neutropenic pediatric patients with acute leukemia receiving chemotherapy, to stratify the risks of febrile neutropenic episodes whether it can have important implication in term of management and outcome, To determine the bacterial agents associated with infection in these episodes, To find out the correlation between outcome and degree of neutropenia.

\section{Patients and Methods}

We prospectively observed patients admitted for treatment of febrile neutropenia to the department of hematology \& oncology in a Childs central teaching hospital / Baghdad, during a period of 10 months (from 1st of March through December 2007). 


\section{Patients}

One hundred seventy two febrile neutropenic episodes occurred in 100 child patients with acute leukemia receiving chemotherapy treatment. The study group of patients may have one episode, two, three or even more febrile episodes are included in this study Patients enrolled in the study underwent complete physical examination, medical history was obtained and baseline laboratory and radiological investigation were performed.

Laboratory methods: As primary evaluation and before starting antibiotic therapy, $(2 \mathrm{mi})$ of Blood samples were obtained by vein puncture after preparation skin with antiseptic iodine and alcohol Samples inoculated in brainheart broth and cultured on blood MacConkey agar under aerobic condition only. Other clinical samples (throat, wound, ear) swabs in addition to urine and stool samples were collected according to patients state, and subjected for routine culture for bacteria. Aspirate for culture from any accessible sites suggesting infection were also taken. Renal and liver function testes were done for general evaluation on admission and repeated with interval according to the disease state. Complete blood count (CBC) and differential count was made every 3 days to follow the response. Other laboratory measurement and procedure were resumed if clinically indicated. Empiric antibiotic therapy was administered (third generation cephalosporin plus gentamycin in most of cases), and All antibiotics were administered intravenously.
Statistical analysis: Chi-square test was applied to determine the Relative importance of various variables.

P-value $<0.05$ was considered as statistically Significant.

\section{Tables and results}

Table below shows the demographic characteristics of 100 children with acute leukemia receiving chemotherapy with 172 febrile neutropenic episodes.

Table 1: Demographic characteristics of patients.

\begin{tabular}{|c|c|c|}
\hline Demographic character & No. of patients & Percentage \\
\hline \multicolumn{3}{|c|}{ Gender } \\
\hline Male & 68 & 68 \\
\hline Female & 32 & 32 \\
\hline \multicolumn{3}{|c|}{ Age (years) } \\
\hline Range & 4 mo. - 14 years \\
\hline Average & $5.9+1.2$ & \\
\hline Mean Number Episodes \\
\hline 1 episode & 40 \\
\hline 2 episodes & 49 & 40 \\
\hline 3 episodes & 10 & 10 \\
\hline > 3 episodes & 1 & 1 \\
\hline
\end{tabular}

Acute lymphoblastic leukemia (ALL) was the most common type of leukemia and therefore the high percentage of episodes.

Table 2: Relation between the type of leukemia and number of febrile episodes.

\begin{tabular}{|c|c|c|}
\hline Type of malignancy & No. of patients (\%) & No. of episodes (\%) \\
\hline ALL & $74(74)$ & $135(78)$ \\
\hline AML & $26(26)$ & $37(22)$ \\
\hline Total & $100(100)$ & $172(100)$ \\
\hline
\end{tabular}

Table below divided the neutropenia in to (Mild, moderate and severe) according to Absolute neutrophil count (ANC).

Table 3: distribution of episodes according to the degree of severity of neutropenia

\begin{tabular}{|c|c|c|}
\hline Degree of neutropenia & No. of episodes & \% \\
\hline Mild $(>500)$ & 69 & 40 \\
\hline Moderate $(200-500)$ & 56 & 33 \\
\hline Severe $(<200)$ & 47 & 27 \\
\hline Total & 172 & 100 \\
\hline
\end{tabular}

Table below shows that the clinical signs and symptoms suggestive of infection were evident in $38 \%$ of febrile neutropenic episodes. The respiratory system was the most affected site. The microbiological documented infection were detected in $16 \%(n=27)$ of the episodes. In majority of episodes there were no focus of infection whether clinical or microbiological.

Table 4: Incidence of clinically and microbiologically documented infections.

\begin{tabular}{|c|c|c|}
\hline & No. of episodes & \% \\
\hline Clinically documented infections & 65 & 38 \\
\hline Respiratory system & 30 & 17 \\
\hline Soft tissue & 17 & 10 \\
\hline GIT & 15 & 9 \\
\hline Urinary system & 3 & 2 \\
\hline Absent signs and symptoms of infection & 107 & 62 \\
\hline Microbiologically documented infections & 27 & 16 \\
\hline No Microbiological document of infection & 145 & 84 \\
\hline
\end{tabular}

Out of 237 cultures (172 from blood and 56 from sites other than blood). Positive isolates were detected in 27 cultures only $(11 \%)$ of total cultures G-ve bacilli were the most frequently isolated organisms $63 \%(n=17)$ of positive isolates. Pseudomonas aeruginosa was the most commonly encountered bacterium in positive cultures $37 \%(\mathrm{n}=10)$, and wounds were the most common sites for isolation $26 \%$ $(n=7) . G+v e$ bacteria represented $37 \%$ of positive isolates. Other organisms isolate and their percentage are shown in table (5) below. 
Table 5: Distribution and frequency of isolated bacteria according to the site of culture

\begin{tabular}{|c|c|c|c|c|c|c|c|}
\hline Isolated Organism & Wound swab (\%) & Throat swab (\%) & Ear swab (\%) & Urine (\%) & Stool (\%) & Blood (\%) & Total \\
\hline Pseudomonas aerugo. & $7(26)$ & $2(7.4)$ & $1(3.7)$ & 0 & 0 & 0 & $10(37.1)$ \\
\hline Staph epidermidis & $1(3.7)$ & $2(7.4)$ & $2(7.4)$ & 0 & 0 & 0 & $5(18.5)$ \\
\hline Klebsiella a spp. & $2(7.4)$ & $1(3.7)$ & 0 & $2(7.4)$ & 0 & 0 & $5(18.5)$ \\
\hline Strept. Pneumoniae & 0 & $3(11.1)$ & $2(7.4)$ & 0 & 0 & 0 & $5(18.5)$ \\
\hline E. coli & 0 & 0 & 0 & 0 & $2(7.4)$ & 0 & $2(7.4)$ \\
\hline Total & $10(37.1)$ & $8(29.6)$ & $5(18.5)$ & $2(7.4)$ & $2(7.4)$ & 0 & $27(100 \%)$ \\
\hline
\end{tabular}

Table below shows the percentage of prolonged fever and neutropenia ( $>1$ week) documented at hospital, and idea about the risk of patients for morbidity and mortality.

Table 6: Duration of fever and neutropenia during episodes.

\begin{tabular}{|c|c|c|}
\hline & No. episodes & $\%$ \\
\hline \multicolumn{3}{|c|}{ Duration of fever } \\
\hline$<1$ week & 131 & 76 \\
\hline$>1$ week & 41 & 24 \\
\hline \multicolumn{2}{|c|}{ Duration of neutropenia } \\
\hline$<1$ week & 117 & 68 \\
\hline$>1$ week & 55 & 32 \\
\hline
\end{tabular}

Table below shows that, the case fatality rate was $24 \%(\mathrm{n}=$ 24) of total patients with febrile neutropenia.

With a significant mortality in severe neutropenicepisodes than in moderate and mild

Table 7: Relationship between mortality rate and degree of neutropenia among neutropenic cancer patients.

\begin{tabular}{|c|c|c|c|c|c|c|c|}
\hline Degree of neutropenia & Mild & & Moderate & Severe & Total \\
\hline & No. & $\%$ & No. & $\%$ & No. & $\%$ & \\
\hline No. of episodes & 69 & 40 & 56 & 33 & 47 & 27 & 172 \\
\hline No. pf death & 3 & 4 & 6 & 11 & 15 & 32 & 24 \\
\hline
\end{tabular}

Chi-square $=12.99 \mathrm{p}$-value $=0.0015$ (highly significant)

\section{Discussion}

With cancer chemotherapy, infectious complications are life threatening and may limit the benefit of antineoplastic therapy.

In this study the frequency of occurrence of fever and neutropenia in leukemic patients was similar to that reported by Stabell $\mathrm{N}$ et al., ${ }^{[6]}$, Paganini HR. et al. ${ }^{[7]}$ and Majd Z et al. ${ }^{[8]}$ That the most common underlying malignant disease was Acute lymphoblastic leukemia (ALL) and so the frequency of febrile neutropenicepisodes. Clinical signs or symptoms of infection, except for fever in febrile neutropenic patients with malignancies were documented by EL- Maghraby SM. et al. ${ }^{[9]}$ in $45.9 \%$ of their study. Haider M. et al. ${ }^{[5]}$. reported clinically documented infection in $39 \%$ of their study. In study carried out by Paganini HR. et al. ${ }^{[7]}$, clinically documented infection was $47 \%$.

In this study clinical signs or symptoms of infection were documented in $38 \%$ of febrile neutropenicepisodes and the respiratory system was the most common site of infection encountered in $17 \%$ of episodes, similar to Haider M. et al. ${ }^{[5]}$. found $17 \%$ on his study.

In this study microbiological documented infection was $16 \%$ of episodes, G-ve organisms were the most frequently isolated in $63 \%$, followed by $\mathrm{G}+\mathrm{ve}$ organisms in $37 \%$ of positive isolates, and this probably due to lack of Centeralacces device in our center.

Hammerstrom J. et al. ${ }^{[10]}$, found that G-ve bacteria represent $54 \%$ of bacteremia in acute leukemia patients with severe neutropeia. Haider M. et al. ${ }^{[5]}$, reported a microbiological documented infection in $59 \%$ of episodes $\mathrm{G}$ +ve were the most frequently isolates in 54\% and G-ve In $39 \%$ of isolates AL-Maghraby SM. et al. ${ }^{[9]}$, found microbiological documented infection in febrile neutropenic children with hematological malignancies was $69 \%$ local sites of infection in $45 \%$ and baccteremia in $24 \%$ ), the $G$ +ve were the predominant organisms.

In this study bacteremia couldn't be detected in any sample. in contrary to all other studs where bacteremia detected in a percentage ranging from $10-30 \%$ KocakU. et al. ${ }^{[11]}$, found that $18 \%$ of febrile neutropenic children with leukemia had bacteremia, and the $\mathrm{G}+\mathrm{ve}$ was the predominant organisms. Mahmood S et al. ${ }^{[12]}$, found a total of $18.8 \%$ o fneutropenic episodes associated with cancer were accompanied by positive blood cultures and $\mathrm{G}+\mathrm{ve}$ was predominant. The high percentage of episodes with no clinical normicrobiological evidence of infection agrees with what found by Castagnola E. et al. ${ }^{[11]}$, who found that the most frequent clinical diagnosis in febrile episodes during chemotherapy induced neutropenia in children was fever of unknown origin in $79 \%$ followed by bacteremia in $10 \%$. Kocak U. et al. ${ }^{[11]}$, found that fever of unexplained origin was seen in $73 \%$ of febrile neutropenic episodes, bacteremia found in $18 \%$. However the use of more sophisticated techniques for isolation of causative microorganisms may lower this percentage.

In this study the case Fatality Rate of $24 \%$ in comparison with other studies was high, and highly significant death rate in severe neutropenic episodes was an alarming outcome.

Haider M. et al. ${ }^{[5]}$, reported (FR) of $3 \%$ of their study, Stabell N. et al. ${ }^{[6]}$, found a mortality rate of $1 \%$. Basus K. et al. ${ }^{[15]}$, reported a mortality rate of $3 \%$ Hamalainen S. et al. ${ }^{[14]}$, reported $11 \%$ died due to severe sepsis. Majid Z. et al. ${ }^{[8]}$, found that the mortality rate in sever neutropenia was $44 \%$ Kocak U. et al. ${ }^{[11]}$ found the fatality rate was zero in febrile neutropenic patients with malignancy.

The prompt initiation of effective empirical antimicrobial therapy, availability of supportive care and better knowledge of microbiology of infection of these patients, the institution and probably in update changing i empincal treatment made the overall mortality among cancer patients in developed countries significantly lower than observed in this study. In our oncology department the choice of antibiotics depend on availabilities of drugs but does not depend on the ideal combination for Coverage of most common pathogen associated with infection in these groups of patients which are (ceftazidime plus vancomycin or carbencillin plus gentamycin), The availabilities and the cost of these druas make the combinat- ion of (ceftriaxone plus garamycin) is the our choice in spite of it is weak action against P.aeruinosa or Staph. Epidermidis, and for this reason $49 \%$ of episodes in this study required modification of empirical antibiotic treatment, in contrary to 
Kocak U et al. ${ }^{[11]}$ and Hammerstrom $\mathrm{J}$ et al. ${ }^{[10]}$, both founds that only $25 \%$ needs modifica- tion of their empirical antibiotic treatment. The difficulty of getting blood and its products, Unavailability of systemic broad spectrum antifungal treatment, Unavailability of supportive medication like hemopoietic growth Factor although it debatable, Late presentation of our patients may be due to poor socio- economic status, bad security conditions or due to difficult transportation. All can be added as additional factors for high mortality.

\section{Conclusions}

Gram-negative bacteria was the most common encountered in positive cultures from local sites other than blood, and wounds swab was the most common site of isolation, Negative blood culture does not exclude presence of bacteremia, There is obvious increase in mortality rate among severely neutropenic patients.

\section{References}

1. Pizzo PA, Rubin M et al. The child with cancer and infection. Empiric therapy for fever and neutropenia, and prevention strategies. J Pediatr. 1991; 119:679.

2. Pizzo PA. Fever in immuno compromised patienis. E Engl. J Med. 1999; 341:893-9000.

3. Hughes et al. Guidelines for the use of antimicrobial agents in neutropenic patients with cancer. CID, 2002, 34.

4. Giamorellou H, Antoniadou A. infectious complication of febrile leucopenia. In fec. Dis Clinics of N. AM. 2001; 15:457-482.

5. Haider M, Ibrahim A, Balawi et al. Fever and granulocytopenia in children with acute lymphoblastic leukemia under chemotherapy. Saudi Med J 2001; 22:423-427.

6. Stabell N, Nordal E, Stensvold E et al. Febrile neutropenia in maren with cancer; A retrospective Norwegian multi centre study of Clinical and microbiological Outcome, Scand J In fec Dis. 2007; 4:1-7.

7. Paganini HR, Rodriguze Z, Zubizarreta $\mathrm{P}$ et al. criteria of low risk of mortality in children with neutropenia and fever during cancer chemotherapy. Medicina (B Aires). 2001; 61:63-6.

8. Majid et al. Blood culture in febrile and neutropenic children with neoplastic diseases. Athesis submitted to the Iraqi commission for medical specialization in partial fulfillment of the requirement for the degree of fellowship of the Iragi committee for medical specialization in pediatrics, 2001.

9. EL-Maghraby SM, Moner MM, Ismail MM et al. The diagnostic value of C-reactive protein, interlukin-8, and monocyte Chemtactic protein in risk stratification of febrile neutropenic children with hematologic malignancies. J Pediat Hematol Oncol. 2007; 29:131-6.

10. Hammerstrom J, Jacobsen T. Bacteremia with granulocytopenia, microbiology and antibiotic treatment. Tidsskr nor Laegeforen. 1998; 118:437-5.

11. Kocak U, Rolston K, Mullen CA et al. Fever and neutropenia in children with solid tumors is similar in severity and outcome to that in children with leukemia. Support Care Cancer. 2002; 10:58-64.

12. Mahmmod S, Revesz T, Mpofu C et al. Febrile episodes in children with cancer in United Arab
Emirates. Pediatr Hematol Oncol. 1996; 13:135-42.

13. Castagnola E, Fontana V, Caviqlia I et al. A prospective study on the epidemiology of febrile episodes during chemotherapy-induced neutropenia in children with cancer or after stem cell transplantation. ClinInfec Dis. 2007; 45:1269-304.

14. Hamalainen $\mathrm{S}$, Kuittinen $\mathrm{T}$ et al. Neutropenic fever and sever sepsis in adult acute myeloid leukemia patients receiving intensive chemotherapy; causes and consequences. Leuk Lymph. 2008; 49:495-501.

15. Basus K, Fernandez ID, Fisher SG et al. Length of stay and mortality associated with febrile neutropenia among children with cancer. J Clinico. 2005; 23:795866. 